\title{
Treatment Intensity, not Age, Affects Survival Time of Patients with Advanced Pancreatic Cancer
}

\author{
Jonas Kunkel, Wolfram Bohle, Silja Henseling, Wolfram G. Zoller
}

Department of General

Internal Medicine

and Gastroenterology,

Katharinenhospital Stuttgart, Germany

\author{
Address for correspondence: \\ Jonas Kunkel \\ Department of General \\ Internal Medicine \\ and Gastroenterology, \\ Katharinenhospital \\ Kriegsbergstraße 60, 70174 \\ Stuttgart, Germany \\ jonas.kunkel3@gmail.com
}

\begin{abstract}
Background \& Aims: Despite older-aged individuals accounting for most patients with pancreatic cancer, elderly patients are still underrepresented in the clinical trials. Our study aims to identify treatment differences as well as to analyze survival times in the younger and older patient group.

Methods: We evaluated the data of 97 pancreatic cancer patients ( $72<75$ years; $25 \geq 75$ years) receiving palliative chemotherapy. Age, comorbidity, body mass index (BMI), tumor localization, metastases, carbohydrate-antigen 19-9 (CA19-9) value, number and type of chemotherapeutic agents and treatment regimens used, treatment lines, toxicity and survival time were assessed.

Results: The age groups did not differ in their initial conditions (comorbidity, BMI, tumor characteristics). However, treatment intensity of patients $\geq 75$ years was lower. Elderly patients received significantly fewer different chemotherapeutic agents and therapeutic regimens, therapy lines and fewer combination chemotherapies. Moreover, elderly patients survived significantly shorter ( 7.6 vs. 12.7 months, $\mathrm{p}=0.001$ ). In multivariance analysis, a significant negative influence on survival time was revealed for low therapy intensity ( $\leq 2$ chemotherapeutics, $\leq 2$ therapy lines), but not for age. In addition, therapy discontinuation and underweight were significantly associated with survival time.

Conclusion: Not age per se but lower therapy intensity leads to a shorter overall survival in the elderly patient group.
\end{abstract}

Key words: pancreatic cancer - chemotherapy - elderly patients - palliative therapy - overall survival.

Abbreviations: 5-FU: 5 fluorouracil; BMI: body mass index; CA19-9: carbohydrate antigen 19-9; FOLFIRI: folinic acid, 5-FU, and irinotecan), FOLFIRINOX (folinic acid, 5-FU, irinotecan and oxaliplatin; FOLFOX: folinic acid, 5-FU, and oxaliplatin.

\section{INTRODUCTION}

With $9 \%$, pancreatic carcinoma has the lowest 5-year survival rate of all cancer types; in more than $50 \%$ a metastatic stage is already present at time of diagnosis, which reduces the 5 -year survival rate to $3 \%[1,2]$. The median survival in palliative situations under combination chemotherapy ranges from 7 to 11 months [3-6]. Patients with advanced stages receiving chemotherapy showed a longer overall survival compared to patients being treated with „best supportive care" [7].
Especially in elderly patients, the incidence of pancreatic carcinoma is increasing and is predicted to rise by $73 \%$ between 2010 and 2030 [8]. However, this development towards a continuously aging patient population is not reflected in clinical trials $[3,9,10]$. Hutchins et al. [9] showed that even though $47 \%$ of all cancer patients are $>70$ years old, only $13 \%$ of the patients in cancer studies belong to this age group. This discrepancy was also observed when studies excluding elderly patients were not taken into account [9].

In clinical studies on pancreatic cancer, the median age of study participants only ranges between 58 and 64 years; in some cases, all patients $>75$ years are excluded by study design [ $3-5$, 11]. Since especially elderly patients are not representatively enrolled in trials even though the average age of onset is beyond 70 years, it must be questioned if it is legitimate to generalize study results to the overall population.

The purpose of this study was to identify differences between the younger and older patient group regarding initial 
conditions and chemotherapeutic treatment as well as to evaluate their influence on survival time.

\section{METHODS}

Our study retrospectively analyzed the data of 136 patients who underwent palliative chemotherapy for locally advanced or metastatic pancreatic cancer between January 2011 and March 2018 at the Department of Gastroenterology, Klinikum Stuttgart.

We analyzed the following parameters: age $(</ \geq 75$ years $)$, gender, body mass index (BMI), comorbidities [cardiovascular diseases, diabetes mellitus, kidney diseases (creatinine $>1.5$ $\mathrm{mg} / \mathrm{dl}$ ), lung diseases], carbohydrate antigen 19-9 (CA199) concentration, tumor localization (pancreatic head vs. other localization); distant metastases at initial diagnosis; chemotherapeutic agents used [gemcitabine, nab-paclitaxel, erlotinib, 5 fluorouracil (5-FU), irinotecan, oxaliplatin], therapeutic regimens used [gemcitabine monotherapy, gemcitabine + nab-paclitaxel, 5-FU monotherapy, FOLFOX (folinic acid, 5-FU, and oxaliplatin), FOLFIRI (folinic acid, 5-FU, and irinotecan), FOLFIRINOX (folinic acid, 5-FU, irinotecan and oxaliplatin) regimen], number of different chemotherapeutic agents, therapeutic regimens and therapy lines used, reason for ending a therapy line (progress, toxicity, patient's wish, good response to treatment, death), preplanned treatment discontinuation ( $\geq 6$ weeks) with number, duration and reason (remission, toxicity, patient's wish), dose reduction ( $<75 \%$ dose in $>50 \%$ of administrations), toxicity leading to modification or interruption of chemotherapy (hematological toxicity such as anemia, leukopenia, thrombopenia; nonhematological toxicity such as allergy, polyneuropathy, gastrointestinal toxicity), duration of therapy from first to last administration of palliative chemotherapy as well as survival time from palliative diagnosis to endpoint (death or 01.04.2018).

Exclusion criteria of our study were: treatment initiation after June 2017, different histology than adenocarcinoma of pancreas, additional cancer; curative treatment only, external continuation of chemotherapy, which did not allow a complete follow up. A total of 39 patients were excluded: 11 patients due to other histology, 1 patient due to additional cancer, 10 patients due to incompletely documented history of disease and 17 patients due to external continuation of chemotherapy.

All statistical calculations were performed using IBM SPSS Statistics (Version 25). Age groups were compared using Chi-Square test, Fisher's Exact test, Mann-Whitney U-test and $t$-test. Survival analyses were performed using KaplanMeier procedure and Log-Rank test. Prognostic factors were calculated by Cox regression method, a p-value $<0.05$ was classified as statistically significant. The local Ethics Committee approved the study.

\section{RESULTS}

The study included 97 patients ( 37 female, 60 male), 90 patients died during the observation period, 7 lived beyond the end of data collection. The median age was 67 years (range 38 90 years), with 72 patients $<75$ years (median age 64 years) and 25 patients $\geq 75$ years (median age 78 years). The characteristic of included patients are displayed in Table I.

The most frequently used chemotherapeutic drugs were 5-FU (74\%), gemcitabine (73\%), oxaliplatin (73\%) and irinotecan (60\%); nab-paclitaxel (34\%) and erlotinib (9\%) were used less frequently. 5-FU, oxaliplatin, irinotecan and nab-paclitaxel were used significantly more often in the younger patient group $(\mathrm{p}=0.003, \mathrm{p}=0.001, \mathrm{p}<0.001, \mathrm{p}=0.027)$. In median, the patients received 3 different chemotherapeutic drugs (range 1-6). 79\% of patients $<75$ years received 3-6 different chemotherapeutic agents, in contrast to only $8 \%$ of patients $\geq 75$ years $(\mathrm{p}<0.001$; Table II).

The most frequently used therapeutic regimes were FOLFIRINOX (56\%) and gemcitabine monotherapy (47\%); less frequently the regimes gemcitabine + nab-paclitaxel (34\%), FOLFOX (34\%), 5-FU monotherapy (20\%) and FOLFIRI

Table I. Patients' characteristics

\begin{tabular}{|c|c|c|c|c|c|}
\hline & & $\begin{array}{l}\text { All patients } \\
\text { n (\%) }\end{array}$ & $\begin{array}{c}<75 \text { years } \\
\mathrm{n}(\%)\end{array}$ & $\begin{array}{c}\geq 75 \text { years } \\
\mathrm{n}(\%)\end{array}$ & $\mathrm{p}$ \\
\hline $\mathrm{N}$ & & 97 & 72 & 25 & - \\
\hline Average age (range) & & $66.9(38-90)$ & $62.7(38-74)$ & $79(75-90)$ & - \\
\hline \multirow{2}{*}{ Gender } & female & $37(38)$ & $24(33)$ & $13(52)$ & \multirow[t]{2}{*}{0.098} \\
\hline & male & $60(62)$ & $48(67)$ & $12(48)$ & \\
\hline \multirow{4}{*}{ BMI } & underweight & $7(7)$ & $4(6)$ & $3(12)$ & \multirow{4}{*}{0.102} \\
\hline & normal weight & $65(67)$ & $45(62)$ & $20(80)$ & \\
\hline & overweight & $21(22)$ & $19(26)$ & $2(8)$ & \\
\hline & obesity & $4(4)$ & $4(6)$ & $0(0)$ & \\
\hline \multirow[t]{2}{*}{ Localization } & pancreatic head & $52(54)$ & $42(58)$ & $10(40)$ & \multirow[t]{2}{*}{0.113} \\
\hline & other localization & $45(46)$ & $30(42)$ & $15(60)$ & \\
\hline Distant metastases & & $76(78)$ & $56(78)$ & $20(80)$ & 0.816 \\
\hline \multirow[t]{2}{*}{ Comorbidities } & cardiovascular & $50(52)$ & $36(50)$ & $14(56)$ & 0.605 \\
\hline & diabetes mellitus & $38(39)$ & $27(38)$ & $11(4)$ & 0.566 \\
\hline $\begin{array}{l}\text { Median CA19-9 } \\
\text { concentration }(\mathrm{U} / \mathrm{ml})\end{array}$ & & 743 & 840 & 477.5 & 0.554 \\
\hline
\end{tabular}


(14\%) were used. Gemcitabine monotherapy was administered significantly more often in the older patient group $(\mathrm{p}=0.004)$; FOLFIRINOX and gemcitabine + nab-paclitaxel were used significantly more often in the younger patient group $(\mathrm{p}<0.001$, $\mathrm{p}=0.027$ ). In median, 2 different therapy regimens were used in the overall collective, $39 \%$ of patients $<75$ years received $>$ 2 different regimens, this was observed in only $12 \%$ of patients $\geq 75$ years $(\mathrm{p}=0.013$; Table II).

The median number of therapy lines was significantly lower in the older patient group ( 1 vs. $2.5, \mathrm{p}=0.002$ ); in addition, the therapy of elderly patients was significantly more often based on only 1-2 therapy lines (76\% vs. $50 \%$, $\mathrm{p}=0.025)$. The most frequent reason for ending a therapy line was tumor progression (33\%), followed by toxicity (23.5\%) or death (19.3\%), with no difference between age groups (Table II).

Preplanned treatment discontinuation was performed in 20 patients (11 patients with 1 discontinuation, 9 patients with 2-3 discontinuations). The most frequent reason for interrupting therapy was good response (80\%), toxicity and patient wish each caused about $10 \%$ of the discontinuations. The median duration of a therapy-free interval was 4.4 months. Neither

Table II. Characteristics of chemotherapeutic treatment

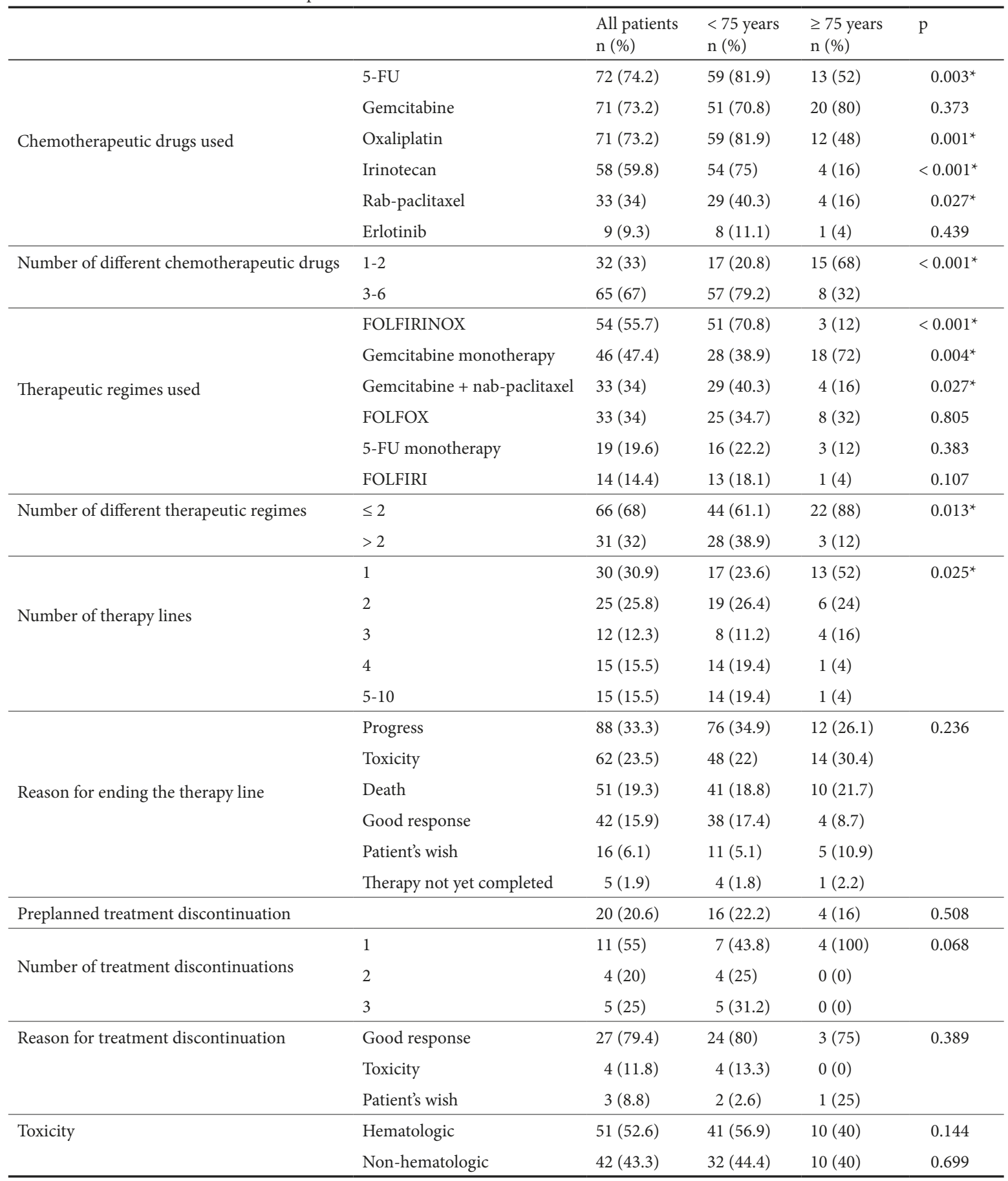

5-FU: 5 fluorouracil; FOLFOX: folinic acid, 5-FU, and oxaliplatin; FOLFIRI: folinic acid, 5-FU, and irinotecan; FOLFIRINOX: folinic acid, 5-FU, irinotecan and oxaliplatin. 
number, reasons nor duration of therapy discontinuations differed between age groups (Table II).

Hematological side effects occurred in $53 \%$ of patients, non-hematological side effects in $43 \%$. The most frequent were gastrointestinal toxicity (30\%) and polyneuropathy (19\%), less frequent allergy (5\%) and cholinergic syndrome (2\%). All adverse events were similar in both age groups (Table II).

Median survival time for the entire population was 9.9 months (range 0.9 - 47.1 months), patients $<75$ years survived significantly longer (12.7 vs. 7.6 months, $\mathrm{p}=0.001$; Fig. 1 ).

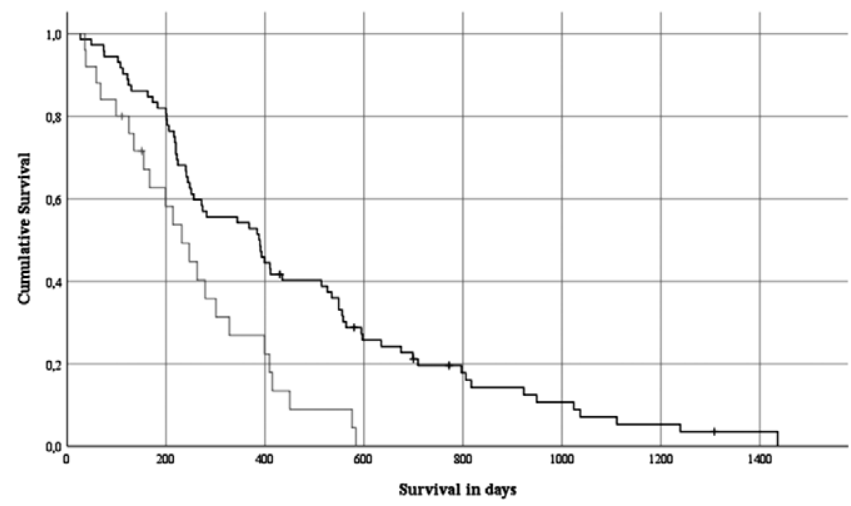

Fig. 1. Kaplan-Meier estimates of survival (in days) by age groups. Median survival of patients $<75$ years (black line) was 12.7 months, compared to 7.6 months for patients $\geq 75$ years (grey line; $p=0.001$ ).

In univariate analysis, survival time was positively affected by the following parameters: age $<75$ years ( $\mathrm{p}=0.001$ ), CA19- 9 $\leq 190 \mathrm{U} / \mathrm{ml}(\mathrm{p}=0.031),>2$ different chemotherapeutic drugs used ( $<<0.001$; Fig. 2 ), $>2$ different therapeutic regimens used $(\mathrm{p}<0.001), 5$-FU monotherapy $(\mathrm{p}=0.001)$, FOLFIRI regime $(\mathrm{p}=0.021)$, FOLFIRINOX regime $(\mathrm{p}=0.001)$, higher number of therapy lines $(p<0.001, p<0.001, p=0.007$; Fig. 3$)$ as well as preplanned therapy discontinuation $(\mathrm{p}<0.001)$. Underweight $(\mathrm{p}=0.029, \mathrm{p}=0.003)$, on the other hand, had a negative effect on survival time (Table III).

Multivariance analysis revealed a positive influence on survival time exclusively for treatment with $>2$ different

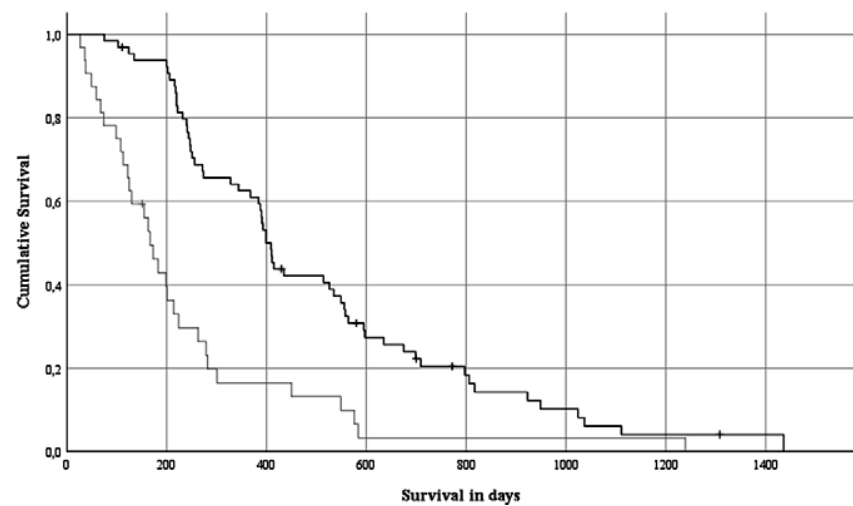

Fig. 2. Kaplan-Meier estimates of survival (in days) by number of different chemotherapeutic drugs used. Median survival of patients treated with $\leq 2$ chemotherapeutic drugs (grey line) was 5.5 months, compared to 13.4 months for patients treated with $>2$ chemotherapeutic drugs (black line; $\mathrm{p}<0,001$ ).

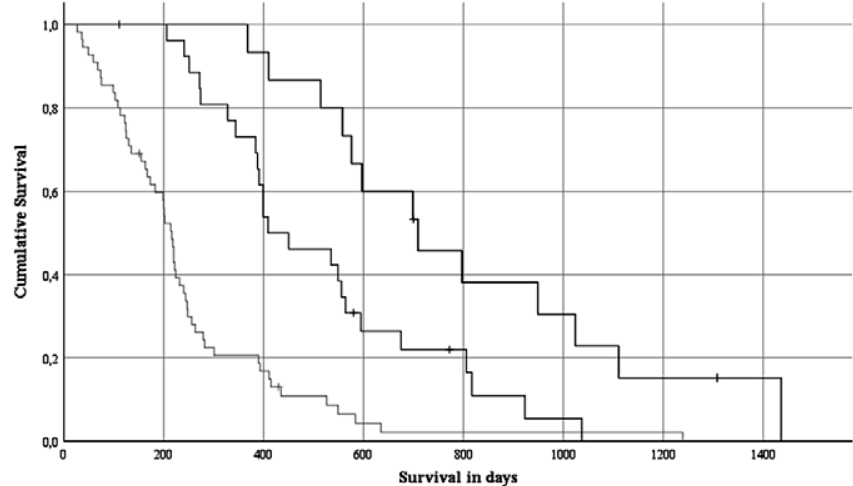

Fig. 3. Kaplan-Meier estimates of survival (in days) by number of therapy lines. Median survival of patients treated with 1-2 therapy lines (light grey line) was 4.1 months, compared to 13.4 months for patients treated with 3-4 therapy lines (dark grey line; $p<0,001$ ). Median survival of patients treated with 1-2 therapy lines was 4.1 months, compared to 23.2 months for patients treated with 5-10 therapy lines (black line; $\mathrm{p}<0,001$ ). Median survival of patients treated with 3-4 therapy lines was 13.4 months, compared to 23.2 months for patients treated with 5-10 therapy lines $(\mathrm{p}=0,007)$.

chemotherapeutic drugs $(\mathrm{p}=0.010)$ and preplanned therapy discontinuation $(\mathrm{p}<0.001)$. Treatment with $1-2$ therapy lines $(\mathrm{p}=0.018)$ as well as underweight $(\mathrm{p}=0.011)$ remained as prognostically negative. In contrast, age was not an independent prognostic factor in the multivariance analysis (Table IV).

\section{DISCUSSION}

In Germany, the average age of onset of pancreatic cancer is 71 years for men and 75 years for women [2]. However, in clinical studies elderly patients are not representatively enrolled. In patients $\geq 65$ years, Talarico et al. [10] and Hutchins et al. [9] observed a discrepancy of 33\% and 35\% respectively, between study participation and the actual proportion of patients within this age group $[9,10]$. The underrepresentation of elderly patients in clinical trials is further aggravated by strict exclusion criteria regarding age, organ function and functional status $[3,12]$.

For advanced pancreatic cancer, survival periods of 7-11 months under combination chemotherapy are described $[3-6,13]$. In our study, the median survival time was 9.9 months. However, the survival in the older patient group was significantly shorter than in the younger patient group (7.6 vs 12.7 months). It is insufficiently described to what extent older age has a negative influence on survival time, as the results are mainly from retrospective studies. Nakai et al. [14] demonstrated that there was no significant difference in survival time between patients $<75$ and $\geq 75$ years [14]. In contrast, Van der Geest et al. [15] described a significantly shorter survival for patients $\geq 75$ years even after adjustment for confounding factors.

All studies mentioned below separated the groups at the age of either 60 or 65 . In 3 of these studies, age did not influence survival time $[6,16,17]$. In contrast, the study by Tas et al. [18] showed that age $\geq 60$ years is a negative prognostic factor, although the age groups were equal regarding 
Table III. Univariate analysis

\begin{tabular}{llcccc}
\hline & & $\begin{array}{c}\text { Median survival } \\
\text { (in months) }\end{array}$ & & $\begin{array}{c}\text { Median survival } \\
\text { (in months) }\end{array}$ & $p$ \\
\hline Age & $<75$ years & 12.7 & $\geq 75$ years & 7.6 & $0.001^{*}$ \\
\hline \multirow{3}{*}{ BMI } & $<18.5 \mathrm{~kg} / \mathrm{m}^{2}$ & 7.6 & $18.5-25 \mathrm{~kg} / \mathrm{m}^{2}$ & 9.9 & $0.029^{*}$ \\
& $<18.5 \mathrm{~kg} / \mathrm{m}^{2}$ & 7.6 & $>25 \mathrm{~kg} / \mathrm{m}^{2}$ & 16.9 & $0.003^{*}$ \\
& $18.5-25 \mathrm{~kg} / \mathrm{m}^{2}$ & 9.9 & $>25 \mathrm{~kg} / \mathrm{m}^{2}$ & 16.9 & 0.128 \\
\hline Comorbidity & yes & 9.8 & no & 9.0 & 0.572 \\
\hline Localization & pancreatic head & 8.9 & other localization & 12.7 & 0.562 \\
\hline Distant metastases & yes & 9.1 & no & 12.8 & 0.264 \\
\hline CA19-9 value & $\leq 190 \mathrm{U} / \mathrm{ml}$ & 11.3 & $>190 \mathrm{U} / \mathrm{ml}$ & 10.8 & $0.031^{*}$ \\
\hline Number of chemo-therapeutic druggs & $1-2$ & 5.5 & $3-6$ & 13.4 & $<0.001^{*}$ \\
\hline Number of therapeutic regimes & $\leq 2$ & 7.3 & $>2$ & 18.2 & $<0.001^{*}$ \\
\hline Gemcitabine monotherapy & yes & 9.9 & no & 9.0 & 0.815 \\
\hline Gemcitabine + nab-paclitaxel & yes & 13.4 & no & 8.1 & 0.060 \\
\hline 5-FU monotherapy & yes & 18.3 & no & 8.1 & $0.001^{*}$ \\
\hline FOLFOX & yes & 13.1 & no & 8.6 & 0.161 \\
\hline FOLFIRI & yes & 18.5 & no & 8.4 & $0.021^{*}$ \\
\hline FOLFIRINOX & yes & 13.4 & no & 6.6 & $0.001^{*}$ \\
\hline \multirow{2}{*}{ Number of therapy lines } & $1-2$ & 7.1 & $3-4$ & 13.4 & $<0.001^{*}$ \\
& $1-2$ & 7.1 & $5-10$ & 23.2 & $<0.001^{*}$ \\
\hline Treatment discontinuation & yes & 13.4 & $5-10$ & 23.2 & $0.007^{*}$ \\
\hline For & & & no & 8.1 & $<0.001^{*}$ \\
\hline
\end{tabular}

For abbreviations see Table II.

Table IV. Multivariate analysis (hazard ratio > 1 represents longer survival)

\begin{tabular}{lccc}
\hline & & $\begin{array}{c}\text { Hazard ratio } \\
(95 \% \mathrm{CI})\end{array}$ & $\mathrm{p}$ \\
\hline Treatment discontinuation & no & 1 & $<0.001$ \\
& yes & 5.95 & \\
Number of chemotherapeutic & $\leq 2$ & 1 & 0.010 \\
drugs & $>2$ & 2.30 & \\
& & $(1.22-4.36)$ & \\
Number of therapy lines & $>2$ & 1 & 0.018 \\
& $\leq 2$ & 0.46 & \\
& & $(0.25-0.86)$ & \\
Underweight & no & 1 & 0.011 \\
& yes & $(0.06-0.55)$ & \\
\hline
\end{tabular}

other parameters such as CA19-9 level, performance status and tumor localization. The collective was treated either chemotherapeutically (mono- or combination therapies) or by „best supportive care” and it was not evaluated whether the age groups received different treatment modalities or intensities. Thus, it remains unclear what influence treatment modality or intensity had on the survival time of the age groups [18]. The randomized trials on erlotinib + gemcitabine by Moore et al. [4] as well as FOLFIRINOX by Conroy et al. [3] also investigated the role of age ( $\leq />65$ years) as a prognostic factor. Moore et al. [4] could not find any influence on survival time; Conroy et al. [3] demonstrated that age of $>65$ years was an independent negative prognostic factor with a hazard ratio of 1.47 .
Generally, we consider a classification of the age groups into under/above 60 or 65 years as unsuitable. Fragility increases continuously with age and is usually observed to a relevant extent only in patients over 75 years of age [19-21]. In the study conducted by Collard et al. [20], a prevalence of fragility of $10 \%$ was observed in 75-79-year-old, and 16\% in 80-84-year-old. In contrast, patients aged 65-69 years had a prevalence of only $4 \%$ [20]. To be able to precisely depict differences between younger and older patients, a separation of the patient cohort at 75 years of age seems more appropriate.

Apparently, patients $\geq 75$ years survived shorter in our study, but age was not a parameter affecting survival time in the multivariance analysis. Consequently, other factors in the older patient group must be present which determine the shorter survival time. Park et al. [22] also observed that age only influenced survival time in the univariance analysis, while initial CA19-9 levels, tumor stage and treatment modality were independent prognostic factors. In contrast to our study, Park et al. [22] did not investigate the differences between the age groups in their treatment and whether such differences explain the shorter survival of elderly patients.

Considering that in our study baseline conditions of the two groups were the same in terms of comorbidity, tumor localization, metastases and CA19-9 value, the reasons for the differences in survival time must be based on treatment modality. Patients $\geq 75$ years were significantly less likely to receive a therapy based on more than 2 different drugs or containing more than 2 therapy lines. These two factors remained prognostically relevant in multivariance analysis, which highlights the influence of treatment intensity on survival time. 
Moreover, elderly patients were significantly less likely to be treated with more than 2 regimens and significantly less likely to be treated with combination regimens such as FOLFIRINOX or gemcitabine + nab-paclitaxel. Li et al. [13] demonstrated in their study on treatment of patients $\geq 75$ years with pancreatic cancer that combination therapies are used very cautiously; only $3 \%$ received FOLFIRINOX, only $1 \%$ received gemcitabine + nab-paclitaxel. Li et al. [13] explain the limited use of gemcitabine + nab-paclitaxel by the fact that, at the time of trial, the Food and Drug Administration had not yet granted approval. Nevertheless, there is no explanation for the fact that FOLFIRINOX was used with such restraint [13].

Since the trial by Conroy et al. [3], FOLFIRINOX represents the nominally most effective therapeutic option for metastatic pancreatic cancer. However, since this study completely excluded patients $>75$ years of age, it is obvious that the lack of evidence leads to a cautious use in the elderly patient group. In addition, a significantly higher incidence of grade $3 / 4$ side effects was observed when using FOLFIRINOX [3]. Similarly, Hoff et al. [5] described significantly higher rates of grade $3 / 4$ toxicity under gemcitabine + nab-paclitaxel compared to gemcitabine monotherapy [5]. The increased frequency of side effects may lead physicians to be restrained in their use of combination therapy on elderly patients.

Furthermore, age is often associated with an increase in comorbidity $[15,23,24]$. Although such an association could not be identified in our study, it is conceivable that a more moderate form of treatment is chosen due to the comorbidities in elderly patients. Our study showed no influence of comorbidity on survival time. In contrast, a study by Kryzkanowska et al. [25], which also included patients not receiving any chemotherapy, demonstrated a negative influence of comorbidity on survival. Thus, comorbidity seems to shorten survival, especially when it prevents or complicates access to chemotherapeutic treatment.

Treatment intensity was demonstrably lower in elderly patients enrolled in our study. Survival analysis revealed that treatment with combination chemotherapy as well as a higher number of chemotherapeutic agents, therapy regimens and therapy lines extended survival time. Prospective randomized trials on advanced pancreatic cancer have shown that patients treated with combination chemotherapy survived significantly longer [3-5]. In our study, longer survival time was also observed with a higher therapy intensity. Nevertheless, it should be considered that, in general, a longer survival period increases the time in which therapy can be administered. For our study it cannot be clarified to what extent the therapy intensity influenced survival time or whether longer survival generally made it possible to use a larger number of regimens, cytostatics and lines, resulting in a higher therapy intensity.

In our study, survival time was increased when patients received the therapeutic regimes FOLFIRINOX, FOLFIRI or 5-FU monotherapy. Due to the effectiveness of Folfirinox, a survival benefit was expected for patients treated with this regime $[3,26,27]$. It was not expected that the comparatively mild 5-FU monotherapy also extended survival time by 10.2 months. Probably most patients treated with 5-FU monotherapy were those who already had previously received
FOLFIRINOX and responded well. 5-FU monotherapy was then applied with the intention of de-escalation as maintenance therapy. Gemcitabine, on the other hand, which has been described in literature as being more effective than 5-FU [28], was used in our study more frequently in patients for whom aggressive combination therapy was not an option. This might be the reason why gemcitabine monotherapy did not provide such a survival benefit as observed with 5-FU monotherapy.

Finally, in multivariance analysis, therapy discontinuation, underweight and treatment intensity remained as independent prognostic factors. Preplanned therapy discontinuation was the strongest independent prognostic factor. The reason for this considerable survival advantage was naturally not the discontinuation itself but can rather be explained by good response to therapy. Treatment was discontinued in $80 \%$ due to good response, in only $10 \%$ due to toxicity or patient wish. Being underweight had a negative influence on survival time, which shows the relevance of good nutritional status for prognosis. In addition, Inal et al. [29] reported that weight loss has a negative effect on survival. A low treatment intensity in terms of treatment based on $\leq 2$ therapy lines or $\leq 2$ chemotherapeutic drugs turned out to be an independent negative prognostic factor. The previous comparison of the two age groups demonstrated that patients $\geq 75$ years of age were treated significantly more often with a lower intensity.

The limitations of our study are the retrospective and unicentric study design, as well as the small number of elderly patients. The strength of this study is that a complete follow-up was possible for almost all patients, only 7 patients living at the end of data collection could not be completely evaluated.

Evidence-based decision-making in terms of chemotherapeutic treatment of elderly patients is generally difficult due to the lack of randomized therapy studies in this specific age group. Treatment selection usually depends on the subjective assessment of the attending physician. Geriatric assessment offers the possibility of objectivizing therapy effectiveness, toxicity and dropout rates [30,31]. This approach is pursued by the not yet completed phase 4 study of Betge et al. [32] on the use of gemcitabine with or without nab-paclitaxel in elderly patients with pancreatic cancer. The participants are divided into different treatment arms according to their geriatric functional status [32]. These results could provide initial insights into whether stratification based on geriatric assessment is beneficial for the chemotherapeutic treatment of pancreatic carcinoma. These data could lead to further urgently needed prospective studies on this topic.

\section{CONCLUSIONS}

Although elderly patients in our study survived significantly shorter, their chronological age itself had no independent influence on survival time. It has been shown that lower treatment intensity in patients $\geq 75$ years of age has a far greater influence on survival time than age per se.

Conflicts of interest: None to declare.

Authors' contributions: J.K., W.B. and W.Z. conceived and designed the study. J.K., W.B. collected data, J.K. and S.H. performed the 
statistical analysis. J.K. analyzed data; J.K., W.B. and S.H. drafted the manuscript. All authors critically revised the manuscript, approved the final version to be published, and agree to be accountable for all aspects of the work.

\section{REFERENCES}

1. Siegel RL, Miller KD, Jemal A. Cancer statistics, 2018. CA Cancer J Clin 2018;68:7-30. doi:10.3322/caac.21442

2. Kaatsch P, Spix C, Katalinic A, et al. Krebs in Deutschland 2011/2012. 2015. doi: $10.25646 / 3174$

3. Conroy T, Desseigne F, Ychou M, et al. FOLFIRINOX versus gemcitabine for metastatic pancreatic cancer. N Engl J Med 2011;364:1817-1825. doi:10.1056/NEJMoa1011923

4. Moore MJ, Goldstein D, Hamm J, et al. Erlotinib plus gemcitabine compared with gemcitabine alone in patients with advanced pancreatic cancer: a phase III trial of the National Cancer Institute of Canada Clinical Trials Group. J Clin Oncol 2007;25:1960-1966. doi:10.1200/ JCO.2006.07.9525

5. Von Hoff DD, Ervin T, Arena FP, et al. Increased survival in pancreatic cancer with nab-paclitaxel plus gemcitabine. N Engl J Med 2013;369:1691-1703. doi:10.1056/NEJMoa1304369

6. Yi JH, Lee J, Park SH, et al. A prognostic model to predict clinical outcomes with first-line gemcitabine-based chemotherapy in advanced pancreatic cancer. Oncology 2011;80:175-180. doi:10.1159/000328449

7. Sultana A, Smith CT, Cunningham D, Starling N, Neoptolemos JP, Ghaneh P. Meta-analyses of chemotherapy for locally advanced and metastatic pancreatic cancer. J Clin Oncol 2007;25:2607-2615 doi:10.1200/JCO.2006.09.2551

8. Smith BD, Smith GL, Hurria A, Hortobagyi GN, Buchholz TA Future of cancer incidence in the United States: burdens upon an aging, changing nation. J Clin Oncol 2009;27:2758-2765. doi:10.1200/ JCO.2008.20.8983

9. Hutchins LF, Unger JM, Crowley JJ, Coltman CA Jr, Albain KS Underrepresentation of patients 65 years of age or older in cancertreatment trials. N Engl J Med 1999;341: 2061-2067. doi:10.1056/ NEJM199912303412706

10. Talarico L, Chen G, Pazdur R. Enrollment of elderly patients in clinical trials for cancer drug registration: a 7-year experience by the US Food and Drug Administration. J Clin Oncol 2004;22:4626-4631. doi:10.1200/ JCO.2004.02.175

11. Taïeb J, Lecomte T, Aparicio T, et al. FOLFIRI.3, a new regimen combining 5-fluorouracil, folinic acid and irinotecan, for advanced pancreatic cancer: results of an Association des Gastro-Entérologues Oncologues (Gastroenterologist Oncologist Association) multicenter phase II study. Ann Oncol 2007;18:498-503. doi:10.1093/annonc/ mdl427

12. Lewis JH, Kilgore ML, Goldman DP, et al. Participation of patients 65 years of age or older in cancer clinical trials. J Clin Oncol 2003;21:13831389. doi:10.1200/JCO.2003.08.010

13. Li D, Capanu M, Yu KH, Lowery MA, Kelsen DP, O’Reilly EM. Treatment, Outcomes, and Clinical Trial Participation in Elderly Patients With Metastatic Pancreas Adenocarcinoma. Clin Colorectal Cancer 2015;14: 269-276.e1. doi:10.1016/j.clcc.2015.05.005

14. Nakai $Y$, Isayama $H$, Sasaki $T$, et al. Comorbidity, not age, is prognostic in patients with advanced pancreatic cancer receiving gemcitabine-based chemotherapy. Crit Rev Oncol Hematol 2011;78:252-259. doi:10.1016/j. critrevonc.2010.05.007
15. van der Geest LG, Haj Mohammad N, Besselink MG, et al. Nationwide trends in chemotherapy use and survival of elderly patients with metastatic pancreatic cancer. Cancer Med 2017;6:2840-2849. doi:10.1002/cam4.1240

16. Ishii H, Okada S, Nose H, Yoshimori M, Aoki K, Okusaka T. Prognostic factors in patients with advanced pancreatic cancer treated with systemic chemotherapy. Pancreas 1996;12:267-271. doi:10.1097/00006676199604000-00009

17. Vickers MM, Powell ED, Asmis TR, et al. Comorbidity, age and overall survival in patients with advanced pancreatic cancer-results from NCIC CTG PA. 3: a phase III trial of gemcitabine plus erlotinib or placebo. Eur J Cancer 2012;48:1434-1442. doi:10.1016/j.ejca.2011.10.035

18. Tas F, Sen F, Keskin S, Kilic L, Yildiz I. Prognostic factors in metastatic pancreatic cancer: Older patients are associated with reduced overall survival. Mol Clin Oncol 2013;1:788-792. doi:10.3892/mco.2013.131

19. Clegg A, Young J, Iliffe S, Rikkert MO, Rockwood K. Frailty in elderly people. Lancet 2013;381:752-762. doi:10.1016/S0140-6736(12)62167-9

20. Collard RM, Boter H, Schoevers RA, Oude Voshaar RC. Prevalence of Frailty in Community-Dwelling Older Persons: A Systematic Review. J Am Geriatr Soc 2012;60:1487-1492. doi:10.1111/j.15325415.2012.04054.x

21. Song X, Mitnitski A, Rockwood K. Prevalence and 10-Year Outcomes of Frailty in Older Adults in Relation to Deficit Accumulation. J Am Geriatr Soc 2010;58:681-687. doi:10.1111/j.1532-5415.2010.02764.x

22. Park JK, Yoon YB, Kim YT, Ryu JK, Yoon WJ, Lee SH. Survival and prognostic factors of unresectable pancreatic cancer. J Clin Gastroenterol 2008;42:86-91. doi:10.1097/01.mcg.0000225657.30803.9d

23. Coresh J, Astor BC, Greene T, Eknoyan G, Levey AS. Prevalence of chronic kidney disease and decreased kidney function in the adult US population: Third National Health and Nutrition Examination Survey. Am J Kidney Dis 2003;41:1-12. doi:10.1053/ajkd.2003.50007

24. Kearney PM, Whelton M, Reynolds K, Muntner P, Whelton PK, He J. Global burden of hypertension: analysis of worldwide data. Lancet 2005;365:217-223. doi:10.1016/S0140-6736(05)17741-1

25. Krzyzanowska MK, Weeks JC, Earle CC. Treatment of locally advanced pancreatic cancer in the real world: population-based practices and effectiveness. J Clin Oncol 2003;21:3409-3414. doi:10.1200/ JCO.2003.03.007

26. Berger AK, Haag GM, Ehmann M, Byl A, Jäger D, Springfeld C. Palliative chemotherapy for pancreatic adenocarcinoma: a retrospective cohort analysis of efficacy and toxicity of the FOLFIRINOX regimen focusing on the older patient. BMC Gastroenterol 2017;17:143. doi:10.1186/ s12876-017-0709-3

27. Gunturu KS, Yao X, Cong X, et al. FOLFIRINOX for locally advanced and metastatic pancreatic cancer: single institution retrospective review of efficacy and toxicity. Med Oncol 2013;30:361. doi:10.1007/s12032012-0361-2

28. Burris HA 3rd, Moore MJ, Andersen J, et al. Improvements in survival and clinical benefit with gemcitabine as first-line therapy for patients with advanced pancreas cancer: a randomized trial. J Clin Oncol 1997;15:2403-2413. doi:10.1200/JCO.1997.15.6.2403

29. Inal A, Kos FT, Algin E, et al. Prognostic factors in patients with advanced pancreatic cancer treated with gemcitabine alone or gemcitabine plus cisplatin: retrospective analysis of a multicenter study. J BUON 2012;17:102-105.

30. Aaldriks A, Maartense E, Le Cessie S, et al. Predictive value of geriatric assessment for patients older than 70 years, treated with chemotherapy. Crit Rev Oncol Hematol 2011;79:205-212. doi:10.1016/j. critrevonc.2010.05.009 
31. Aparicio T, Bouché O, Francois E, et al. Geriatric analysis from PRODIGE 20 randomized phase II trial evaluating bevacizumab+ chemotherapy versus chemotherapy alone in older patients with untreated metastatic colorectal cancer. Eur J Cancer 2018;97:16-24. doi:10.1016/j.ejca.2018.03.030
32. Betge J, Chi-Kern J, Schulte N, et al. A multicenter phase 4 geriatric assessment directed trial to evaluate gemcitabine+/- nab-paclitaxel in elderly pancreatic cancer patients (GrantPax). BMC Cancer 2018;18:747. doi:10.1186/s12885-018-4665-2 